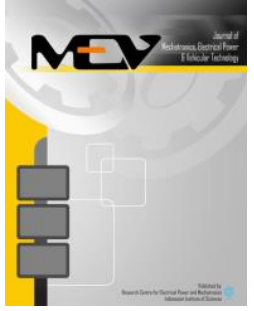

Journal of Mechatronics, Electrical Power, and Vehicular Technology

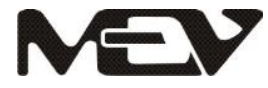

\title{
COMPARATIVE STUDY BETWEEN INTERNAL OHMIC RESISTANCE AND CAPACITY FOR BATTERY STATE OF HEALTH ESTIMATION
}

\author{
M. Nisvo Ramadan, Bhisma Adji Pramana, Sigit Agung Widayat, \\ Lora Khaula Amifia, Adha Cahyadi *, Oyas Wahyunggoro \\ Department of Electrical Engineering and Information Technology, Universitas Gadjah Mada \\ Jalan Grafika no 2 Universitas Gadjah Mada Yogyakarta 55581 Indonesia
}

Received 26 October 2015; received in revised form 27 November 2015; accepted 01 December 2015

Published online 30 December 2015

\begin{abstract}
In order to avoid battery failure, a battery management system (BMS) is necessary. Battery state of charge (SOC) and state of health (SOH) are part of information provided by a BMS. This research analyzes methods to estimate SOH based lithium polymer battery on change of its internal resistance and its capacity. Recursive least square (RLS) algorithm was used to estimate internal ohmic resistance while coloumb counting was used to predict the change in the battery capacity. For the estimation algorithm, the battery terminal voltage and current are set as the input variables. Some tests including static capacity test, pulse test, pulse variation test and before chargedischarge test have been conducted to obtain the required data. After comparing the two methods, the obtained results show that $\mathrm{SOH}$ estimation based on coloumb counting provides better accuracy than $\mathrm{SOH}$ estimation based on internal ohmic resistance. However, the $\mathrm{SOH}$ estimation based on internal ohmic resistance is faster and more reliable for real application.
\end{abstract}

Keywords: battery management system; state of health; lithium polymer; recursive least square; coulomb counting.

\section{INTRODUCTION}

Global warming is one among hot issues a lot of people talk about. In 2013, the average of earth temperature has reached $14.6^{\circ} \mathrm{C}$, it was up to $0.6^{\circ} \mathrm{C}$ compared to the mid-20th century [1]. One of the main causes of global warming is the excessive use of fossil fuel. As it is known number of cars, which are using fossil fuels, continues to grow significantly. This case makes the demand of fossil fuels increases and is known as one major cause of global warming.

Electric car is one way to reduce fossil fuel consumption in order to hold global warming. The use of electric car is estimated to increase rapidly by 2020 [2]. For this car, lithium battery is widely used as the main energy source. Thus battery is key to the success or failure of electric cars. To avoid battery failure, battery management system (BMS) is usually required. BMS is a system aimed for regulating the battery work in its prime area of operation and providing

\footnotetext{
*Corresponding author. Telp : +62-89673487276

E-mail : adha.imam@ugm.ac.id
}

information to the user to perform the necessary actions such as stopping the use of battery or charging the battery. In this case, BMS optimizes the operation of the electric vehicle by knowing the capacity of the battery that has been used as well as ensuring extended battery life. It also controls the cell charging, protects the battery, sets the battery condition, and maintains the balance voltage among battery cells.

It is known that state of charge (SOC) and state of health $(\mathrm{SOH})$ are part of the information provided by BMS. As SOC and $\mathrm{SOH}$ can not be measured directly, an algorithm based on battery model is needed to estimate them. There are various battery models that have been developed. In general, battery model is divided into four classes: physical model (electrochemical), statistical model, analytical model, and electrical equivalent circuit model [3]. Electrical equivalent circuit model is widely used. This model allows battery parameters analysis using mathematical calculations of circuit components [3-5]. Battery in most cases is characterized using parameters of lumped circuit model. With various approaches, 
battery parameters can be found using many algorithms, such as I-ARX [3], and recursive least square [4].

$\mathrm{SOH}$ describes the general condition and performance of the battery compared to that of battery when it is still new. Moreover $\mathrm{SOH}$ estimation allows us to estimate the life of battery to avoid battery failure due to improper usage. On electric cars, $\mathrm{SOH}$ informs the user that the battery replacement is needed when it reaches a certain degradation threshold.

The study about $\mathrm{SOH}$ increases recently. A common approach is considering $\mathrm{SOH}$ estimation as a black box that is solved using artificial intelligence (AI) and machine learning (ML) with various battery condition without considering battery aging mechanism. The studies of AI and ML algorithms include neural network (NN) [5], [6], fuzzy logic [7], and support vector machine (SVM) [8]. In this study, valid and sufficient data are required to provide accurate estimation. Others methods, which are based on numerical analysis, describe battery aging mechanism such as mathematic equation [9], equivalent circuit model [10-12], and electro-chemical model [13].

In this paper, a battery $\mathrm{SOH}$ estimation method is proposed based on internal ohmic resistance and capacity of the battery where performances are compared. As the battery is used, its internal ohmic resistance will change so that it can be used for $\mathrm{SOH}$ estimation. The capacity will degrade due to battery aging that informs the degradation of $\mathrm{SOH}$. The battery capacity can be obtained using coulomb counting method that computes current which flows of the battery.

\section{BATTERY MODEL}

\section{A. Equivalent Circuit Model}

The first-order Resistor-Capacitor (RC) model is one among the best options available to be used in this work. The reason is the trade off between complexity, accuracy, and robustness as stated by X Hu [14]. The capacitance Ccap in Figure 1 represents the SOC of the battery. Using coulomb counting, the SOC can be defined as follows:

$$
S O C=S O C_{o}-\frac{1}{C_{N}} \int_{t o}^{t} \eta I d t
$$

where $\mathrm{SOC}_{0}$ is $\mathrm{SOC}$ at initial time to, $\mathrm{CN}$ is capacity value in standard condition of the battery, $\eta$ is coulombic efficiency that equals 1 while discharge and is smaller than 1 in charge and $I$ represents current which is negative at charge and positive at discharge [15].

In Figure 1, Voc is cell open circuit voltage (OCV) of battery cell, $R_{0}$ is internal ohmic resistance, $\mathrm{Rp}$ is diffusion resistance, $C p$ is diffusion capacitance, Ibatt, $V t$ are the corresponding current and terminal voltage. Here $V t$ is set as output variable. Current source, Ibatt, represents the current flowing out of the battery cell. Ibatt acts as input variable. $R_{0}$ describes internal resistance. The mathematical equations for the equivalent circuit are as follows:

$$
\begin{aligned}
& \dot{u}_{p}=-\frac{u_{p}}{C_{p} R_{p}}+\frac{I_{\text {batt }}}{C_{p}}, \\
& \dot{V}_{o c}=\frac{I_{\text {batt }}}{C_{c a p}} \\
& V_{t}=V_{o c}-u_{p}-I_{\text {batt }} R_{0}
\end{aligned}
$$

where $u_{p}$ is the voltage at the parallel $\mathrm{RC}$ network.

\section{B. Battery Testing and Schedule System}

When a car is moving irregularly, at a certain moment the batteries undergo a large load, but in other time encounter low constant load according to the usage. Changing road conditions can make the battery load varies unpredictably. Thus, this study included a few additional tests to see the battery life as well as to ensure that the battery model is valid. Schematic design of the experiments is shown in Figure 2.
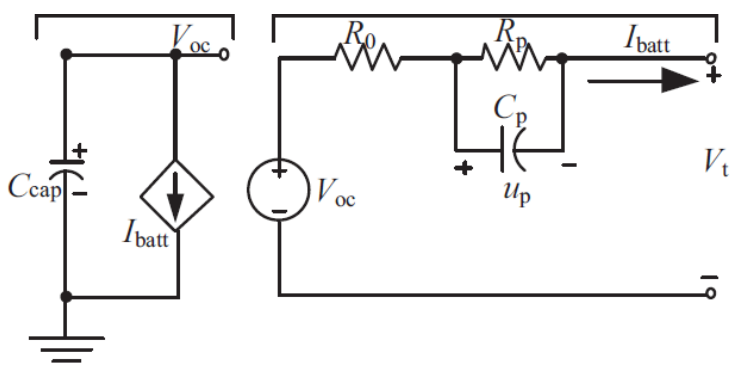

Figure 1. Battery model structure [10]

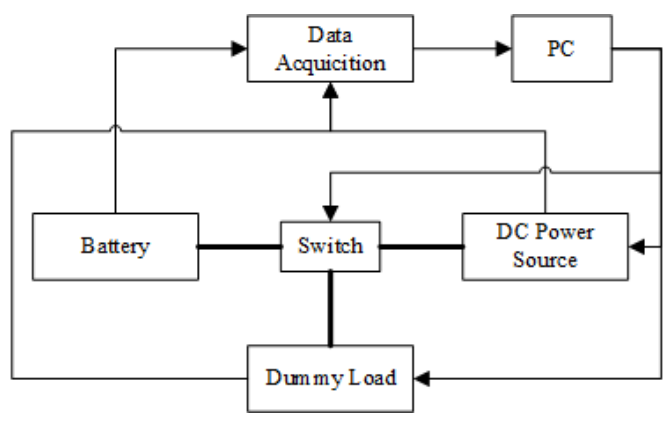

Figure 2. Experimental schematic design 
The objective of this experimental design is to obtain the battery parameter in the form of current and voltage. In this work, the experimental devices consist of DC power source using imax B6 LiPro Balance Charger, dummy load using GW Instek PEL-2004, microcontroller ARDUINO UNO 32, and Matlab® R2013a software for computation and analysis purposes. Moreover, Turnigy Lithium Polymer Battery with a nominal capacity of $2.2 \mathrm{Ah}$ was used in this study. The battery specifications are shown in Table 1 [16].

The experimental procedures are begun with static capacity test at $1 \mathrm{C}$ discharge current as shown in Figure 3. This test is conducted to determine the battery capacity and to obtain the corresponding battery SOC. The next test is pulse test. In this test, the battery is discharged for 30 seconds and then rested for 30 seconds. After that, connect to load again and repeat the proses until the cut off voltage is reached. Cut off voltage is defined as terminal voltage at 20\% SOC. Discharge pulse is 30 seconds for better accuracy of OCV curve because more data points OCV. Rest 30 seconds because at that time the battery terminal voltage has reached steady state. Enlarged rest time will have no effect on the condition of the battery terminal voltage. SOC $20 \%$ is used as discharge limits to avoid over discharge and try to treat the battery as safely as possible.

Pulse test is conducted to identify the battery model parameters of equivalent circuit and to obtain the OCV-SOC relationship. The next test is a test with varying input. The test is used for parameters of equivalent circuit model validation.

The last test is aging cycle test where each cycle is done with constant current charging or
Table 1.

Battery specifications

\begin{tabular}{ll}
\hline Parameter & Value \\
\hline Capacity & $2.2 \mathrm{Ah}$ \\
\hline Max Discharge Current & $44 \mathrm{~A}$ \\
\hline Max Charge Rate Current & $4.4 \mathrm{~A}$ \\
\hline Charge Limit Voltage & $4.2 \mathrm{~V}$ \\
\hline Discharge Limit Voltage & $2.7 \mathrm{~V}$ \\
\hline
\end{tabular}

discharging mode until the voltage reaches a specified value. Battery cycle life is usually determined by the number of cycles of charge discharge. A battery can work well before its nominal capacity falls below $80 \%$ of the initial capacity of the battery [3][9][17]. Therefore, a number of cycles are needed to see the effect that occurs in the battery.

\section{Battery Model Parameter IDENTIFICATION}

\section{A. Identification Method}

Based on the battery model shown in Figure 1, there are four series of parameters that must be obtained, those are $C_{c a p}, R_{o}, R_{p}$, and $C_{p}$. The identification details of these parameters are illustrated as follows:

a) The value of $C_{c a p}$ is obtained from static capacity test as shown in Figure 4.

$$
C_{\text {cap }}=\frac{i \Delta t}{\Delta V}
$$

$b$ ) In the pulse test, $V_{o c}$ is obtained from steady state voltage of each pulse as shown Figure 5.

c) The resistance $\mathrm{R}_{o}$ is proportional to the reduction of voltage drop connected to the load. Resistance $R_{p}$ and capacitance $C_{p}$ are

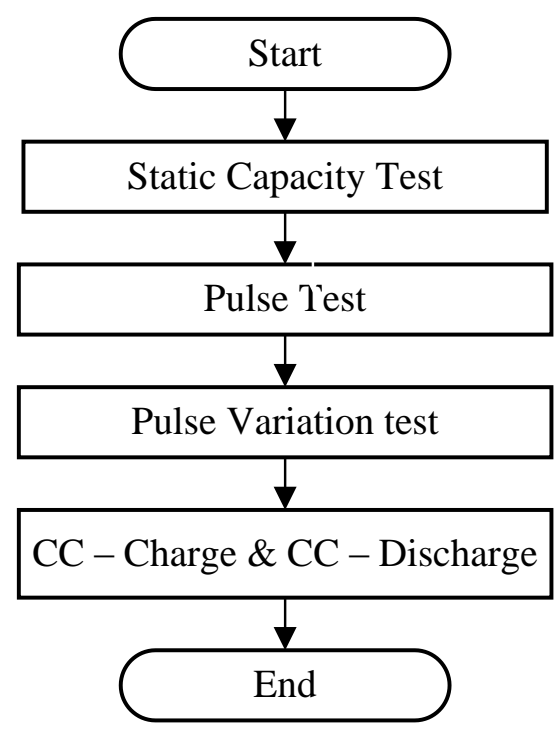

Figure 3. Battery test schedule 
associated with voltage changes as shown in Figure 5. RLS Algorithm is used to obtain the value of $R_{o}, R_{p}$, and $C_{p}$. Linear relationship of input and output is obtained through a transfer function model of the battery.

$V=V_{t}-V_{o c}$

$V_{t}-V_{o c}=\left[\frac{R_{p}}{1+s R_{p} C_{p}}+R_{0}\right] I$

$s=\frac{2}{T} \frac{k-1}{k+1}$

where $\mathrm{T}$ is the sampling period. Then equation (7) can be written as:

$V(k)+a_{1} V(k-1)=b_{0} I(k)+b_{1}(k-1)$

where

$$
\begin{aligned}
& a_{1}=\frac{T-2 R_{p} C_{p}}{T+2 R_{p} C_{p}} \\
& b_{0}=\frac{\left(R_{p}+R_{0}\right) T+2 R_{0} R_{p} C_{p}}{T+2 R_{p} C_{p}} \\
& b_{1}=\frac{\left(R_{p}+R_{0}\right) T-2 R_{0} R_{p} C_{p}}{T+2 R_{p} C_{p}}
\end{aligned}
$$

$a_{1}, b_{0}, b_{1}$ are parameters that need to be solved. In this work, these parameters will be defined using RLS algorithm.

The process in obtaining the parameters using RLS Algorithm is described as follows:

$$
\begin{aligned}
& G(k)=\frac{P(k-1) \varphi(k)}{1+\varphi^{T}(k) P(k-1) \varphi(k)} \\
& \theta(k)=\theta(k-1)+G(k)[V(k)-V(k-1)- \\
& \left.\varphi^{T}(k) \theta(k-1)\right] \\
& P(k)=P(k-1)-G(k) \varphi^{T}(k) P(k-1)
\end{aligned}
$$

where

$$
\begin{aligned}
& \varphi(k)=[V(k-1), I(k), I(k-1)]^{T}, \\
& \theta(k)=\left[-a_{1}, b_{0}, b_{1}\right]^{T} .
\end{aligned}
$$

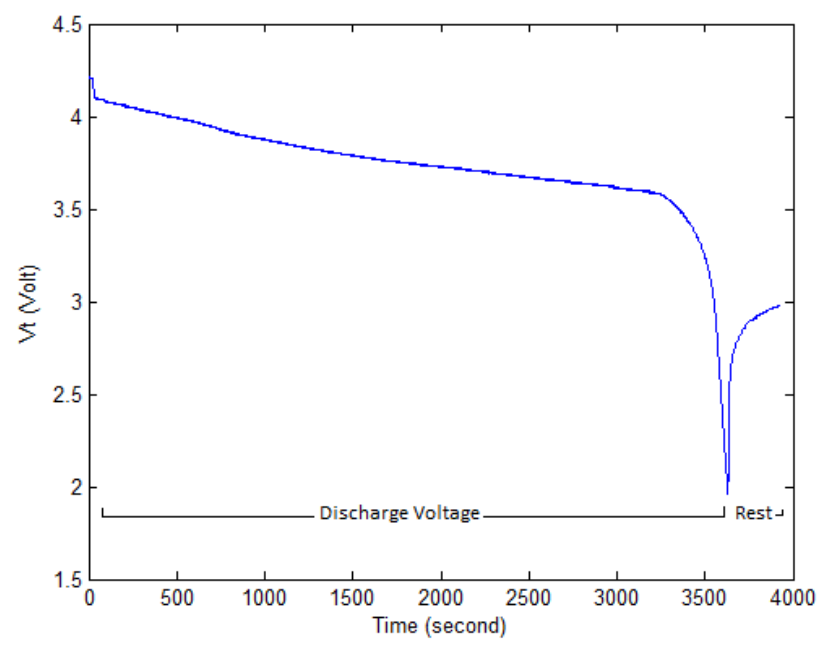

Figure 4. Static capacity test
The initial estimation value of parameter $\theta(0)$ and covariance matrix $\mathrm{P}(0)$ are first determined. $R_{o}, R_{p}$, and $C_{p}$ parameter can be obtained by rewriting equation (10), (11), and (12) as:

$$
\begin{aligned}
& R_{p}=\frac{2\left(a_{1} b_{0}+b_{1}\right)}{1-a_{1}{ }^{2}}, \\
& C_{p}=\frac{T\left(1+a_{1}\right)^{2}}{4\left(a_{1} b_{0}+b_{1}\right)^{\prime}} \\
& R_{0}=\frac{b_{0}-b_{1}}{1+a_{1}} .
\end{aligned}
$$

\section{B. Battery Parameter Estimation Result}

Figure 6 represents the relationship between SOC and OCV. From this relationship, the SOC can be predicted if value of $V_{o c}$ is known. Figure 7,8 , and 9 respectively are $R_{0}-\mathrm{SOC}, R p-\mathrm{SOC}$, and $C p$-SOC curves which are approximated by a second order polynomial.

MATLAB $^{\circledR} /$ Simulink $^{\text {TM }}$ was used to simulate the behavior of the battery. By trial and error, the parameters value are selected at $90 \%$ SOC. This choice will give good estimation errors. It seems that the model is able to follow the changes in the terminal voltage $V_{t}$ as shown in the pulse variations test 1 and 2 in Figure 10(a) and 11(a). In addition, the model is able to predict the battery $V_{t}$ when the current variation is added. It is also indicated by the relative error. In the pulse variation test 1 , the maximum relative error is less than $3 \%$, while in the pulse variation test 2 , the maximum relative error is less than $1.5 \%$ as shown in Figure 10(b) and 11(b).

This research was conducted at room temperature and the battery was not maintained at a certain temperature. However, the battery model can work optimally with the mean relative error for pulse variation of $0.25 \%$ for test 1 and the mean relative error pulse variation of $0.19 \%$ for test 2 .

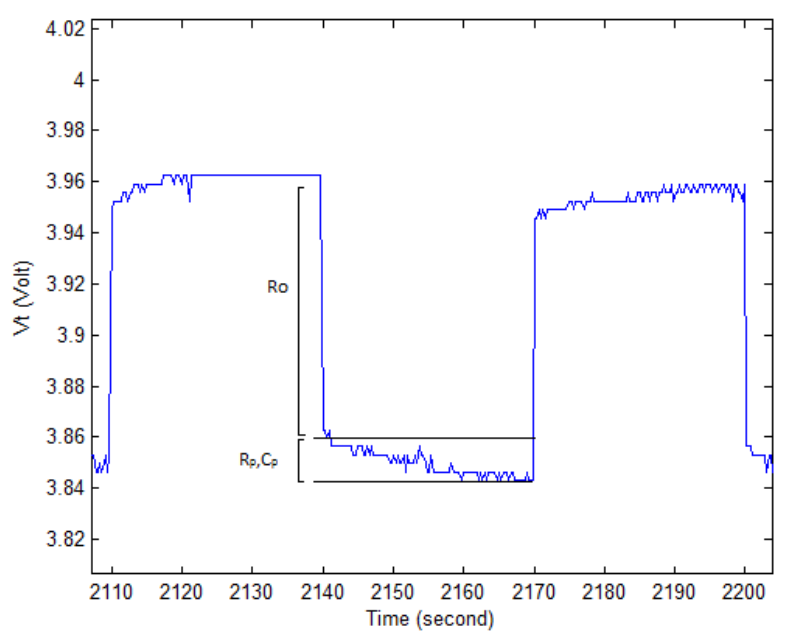

Figure 5. Zoom-in of discharge pulse test 


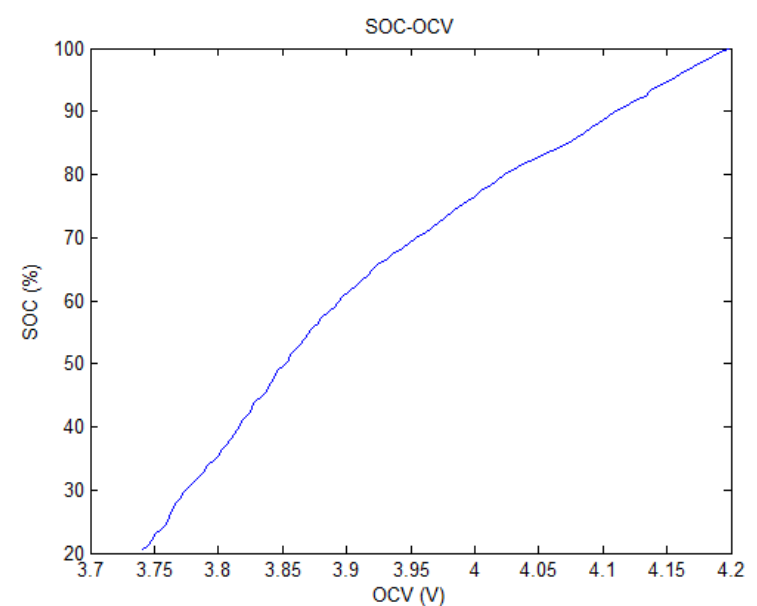

Figure 6. SOC-OCV plot

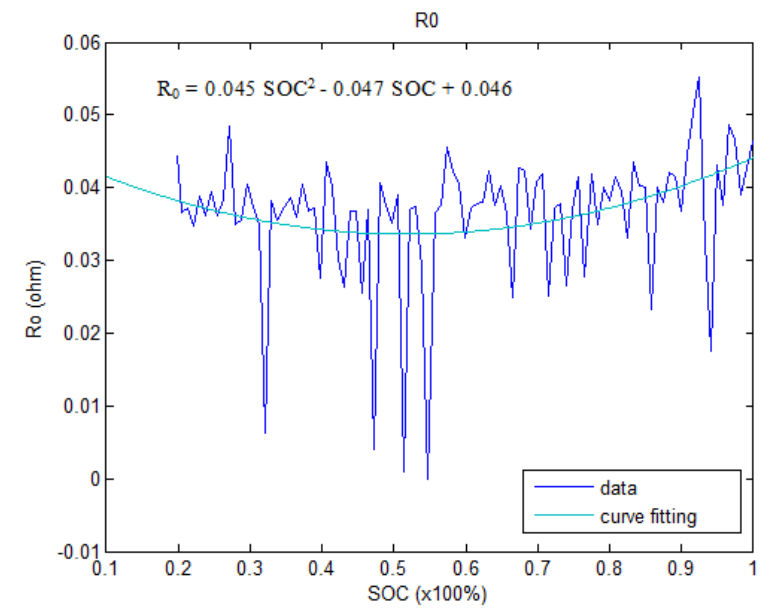

Figure 7. Ro-SOC plot

Based on this error value, this model is acceptable. As shown in Figure 4, the battery discharge curve indicates that the battery is a nonlinear system as the voltage drops drastically when it is nearly depleted. When the battery capacity is approaching its lowest discharged limit, the battery voltage drops quickly and the condition is difficult to be modeled. Hence, the model well describes the battery on the linear part where the SOC is between $80 \%$ and $20 \%$.

\section{STATE OF HEALTh (SOH)}

As the lithium batteries start degrading once manufactured, due to the chemical degradation of the active material and other electrochemical phenomena, the internal ohmic resistance will increase and the capacity will decrease with age [18]. The process of degradation will change the battery performance. By increasing the number of cycles, the battery performance will decrease.

This condition is monitored by $\mathrm{SOH}$. $\mathrm{SOH}$ can be described as the battery performance at the present time compared to the performance at ideal condition and the battery's fresh state [19]. There are many ways to determine the value of $\mathrm{SOH}$, which are based on internal ohmic

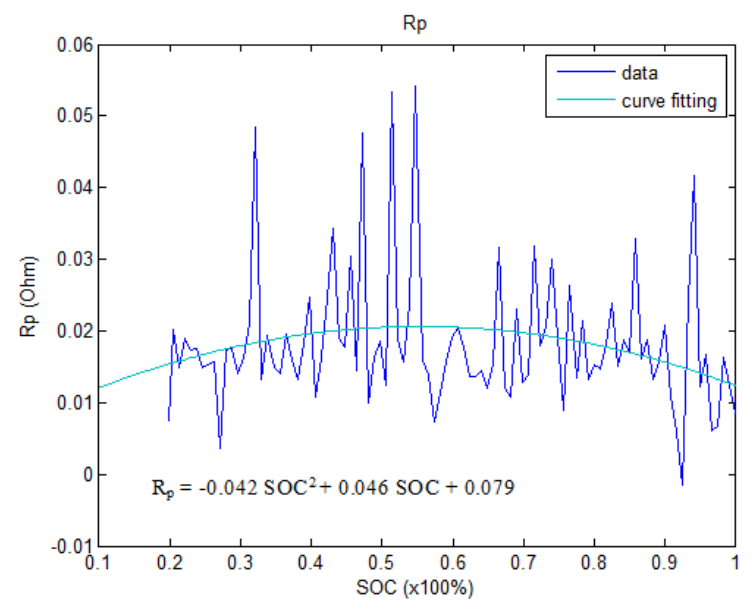

Figure 8. Rp-SOC plot

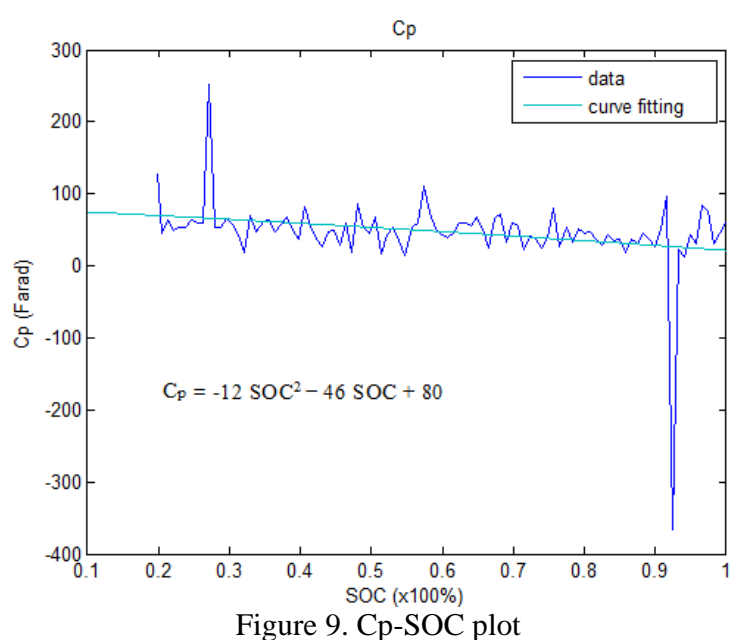

resistance, the battery capacity, the slope of charge or discharge curve, and the curve area of charge or discharge. However, the determination of the $\mathrm{SOH}$ based on internal resistance is more frequently used.

\section{A. SOH Based on Internal Ohmic Resistance}

One of the battery parameters that changes as a result of the degradation process is an internal ohmic resistance. By increasing number of cycles, the battery internal resistance will also increase.

Therefore, the internal resistance can be selected to determine the value of $\mathrm{SOH} .0 \% \mathrm{SOH}$ means that the battery reaches its end of life. A battery reaches its end of life, when the internal resistance rises twice its initial value [17]. In other words:

$$
\begin{array}{lll}
\mathrm{SOH}=100 \% & \Leftrightarrow \mathrm{R}=\mathrm{R}_{I V} \\
\mathrm{SOH}=0 \% & \Leftrightarrow & \mathrm{R}=\mathrm{R}_{E O L}=2 \mathrm{xR}_{I V}
\end{array}
$$

with $R_{I V}$ is the initial value of internal resistance, and $\mathrm{R}_{\mathrm{EOL}}$ is the value of internal resistance at the end of life. Therefore, SOH can be formulated into:

$$
S O H=\left(2-\frac{R}{R_{I V}}\right) \times 100 \% \text {. }
$$




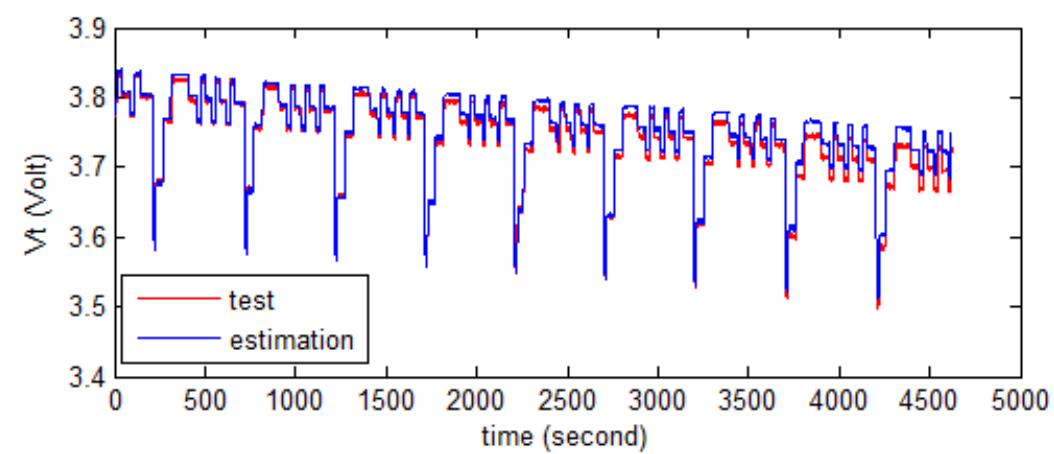

(a)

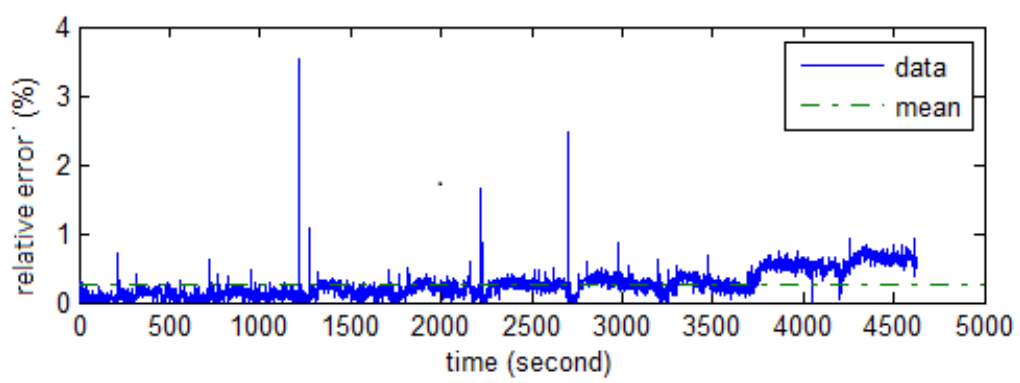

(b)

Figure 10. Validation result in pulse variation test 1; (a) Terminal voltage in pulse; (b) Terminal voltage error in pulse

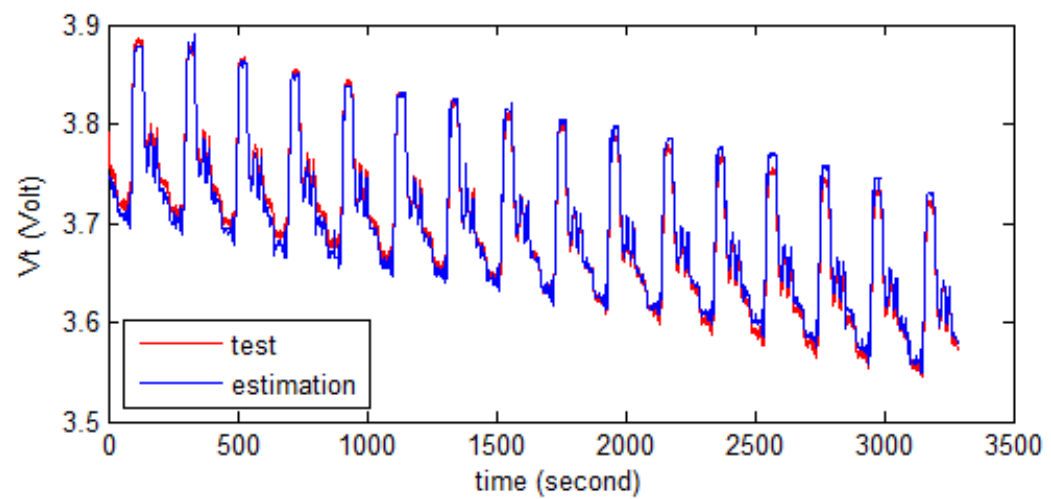

(a)

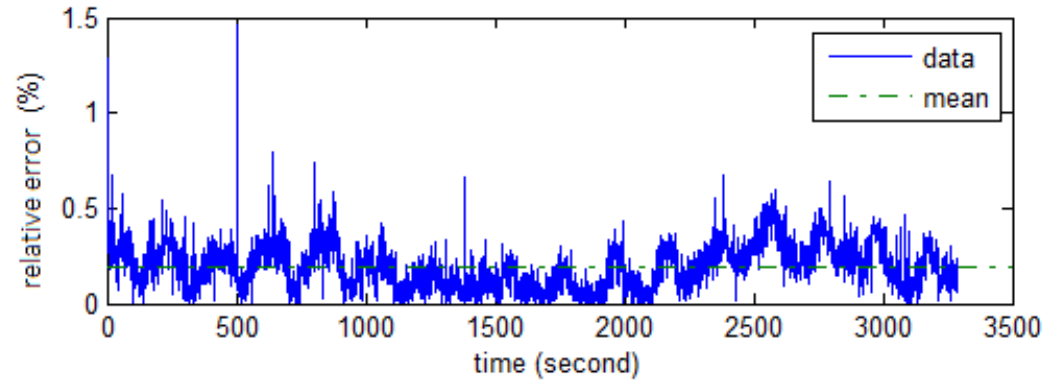

(b)

Figure 11. Validation result in pulse variation test 2; (a) Terminal voltage in pulse; (b) Terminal voltage error in pulse

Other forms of $\mathrm{SOH}$ based on internal resistance is formulated as:

$$
S O H=\left(1+\frac{R_{I V}-R}{R_{I V}}\right) \times 100 \%
$$

where, $R$ is the actual internal resistance and RIV is internal resistance of new battery [20].

The flowchart of SOH estimation on the basis of internal resistance is shown in Figure 12. Data from the experiment is shown in Figure 13. It is seen that the resistance of the battery increases with the increasing number of cycles. SOH curve in Figure 14(a) was $\mathrm{SOH}$ based on internal resistance which was obtained from experiments. Figure 14(b) shows $\mathrm{SOH}$ curve from the battery model, the internal resistance value was obtained from the estimation using RLS Algorithms. 


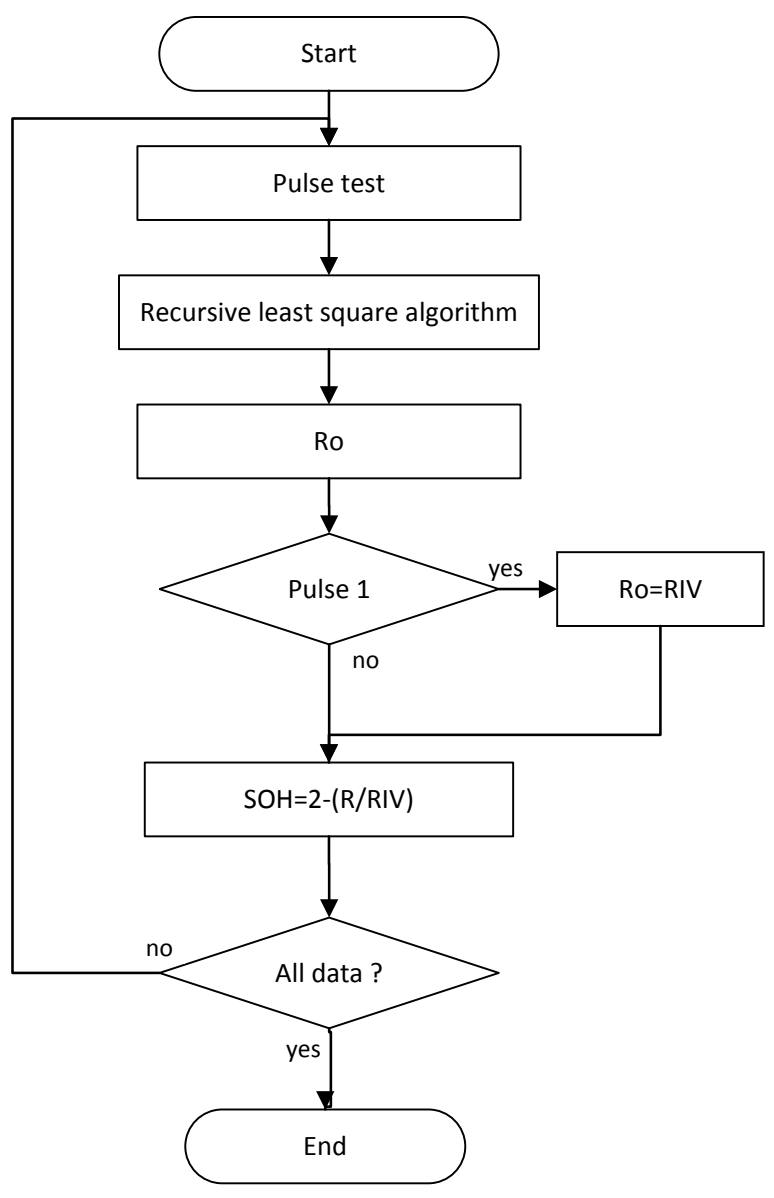

Figure 12. Flowchart of $\mathrm{SOH}$ estimation based on internal ohmic resistance

The result of SOH from the model has similar trend of that from the experimental. When the cycle is more than 20 , the estimated $\mathrm{SOH}$ also shows $0 \%$ which is the same as the experimental results. In addition, the shape of $\mathrm{SOH}$ estimation curve is similar to the $\mathrm{SOH}$ experimental curve. However, this estimation algorithm does not give good estimation accuracy. As shown on the relative error of SOH in Figure 15, it can be seen that the greater error occurs when the cycle is more than 20 .
The SOH estimation based internal ohmic resistance can be determined easily. By obtaining the internal resistance only, the $\mathrm{SOH}$ can be estimated immediately. Therefore, this method is suitable to be applied on electric vehicle.

\section{B. SOH Based on Battery Capacity}

In addition to using the internal ohmic resistance, $\mathrm{SOH}$ of battery can also be determined using its capacity. The battery

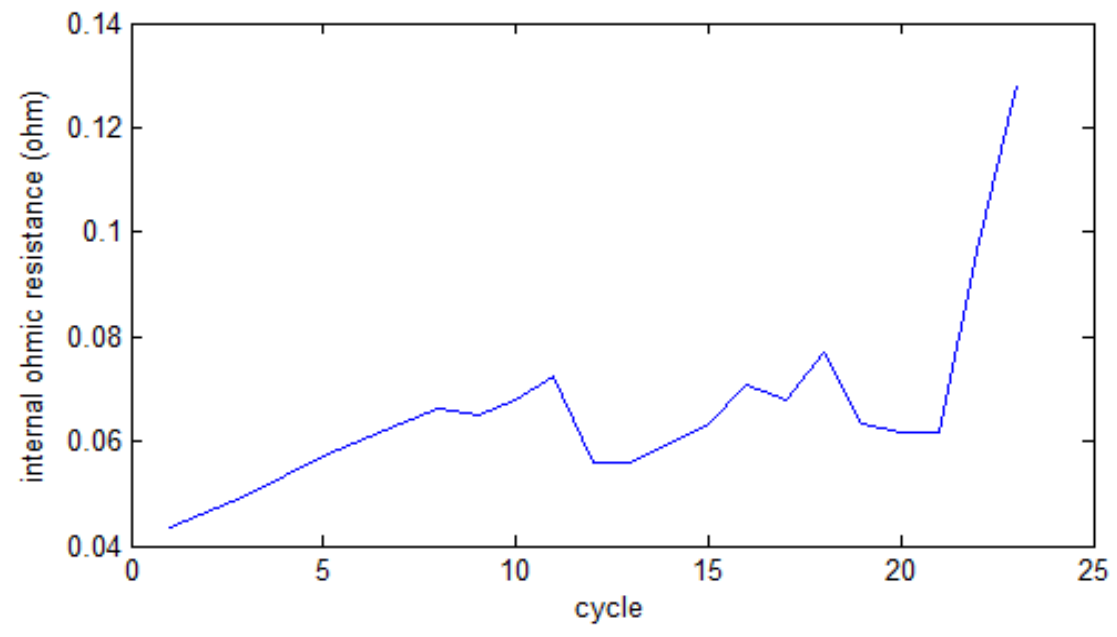

Figure 13. Change of internal ohmic resistance 


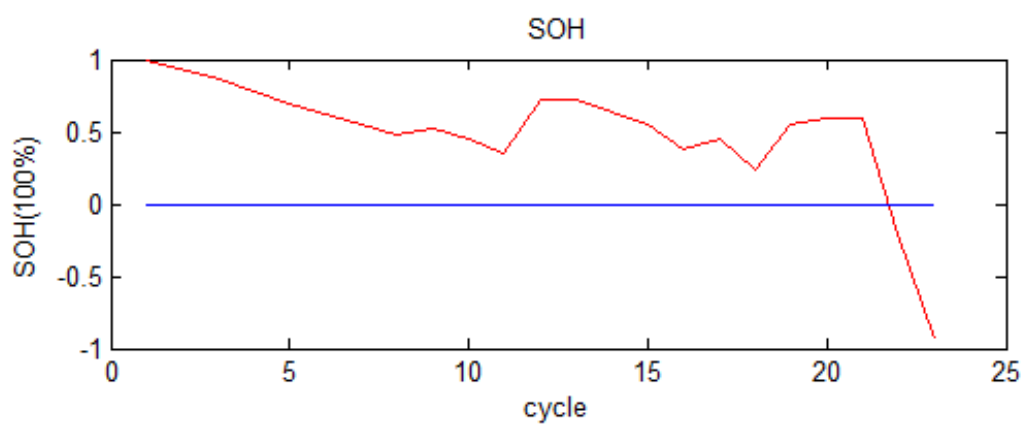

(a)

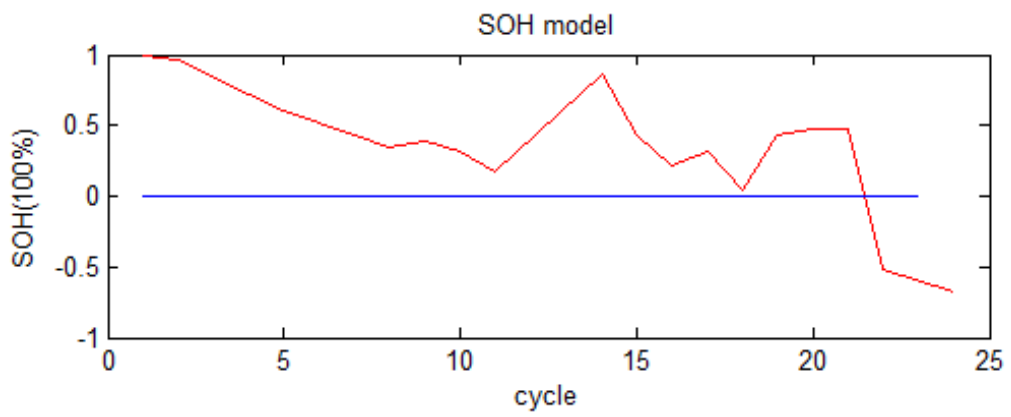

(b)

Figure 14. (a) SOH from experiment; (b) SOH from model

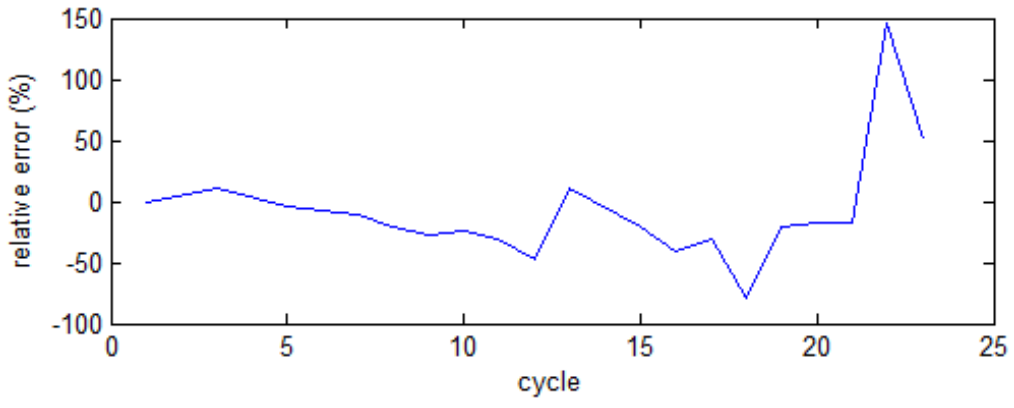

Figure 15. Relative error of SOH

capacity will decrease with increasing number of charge-discharge cycles. The battery reaches its end of life which means $0 \% \mathrm{SOH}$ when the capacity falls to $80 \%$ of its initial value [17]

$$
\begin{array}{lll}
\mathrm{SOH}=100 \% & \Leftrightarrow & \mathrm{C}=\mathrm{C}_{\mathrm{IV}} \\
\mathrm{SOH}=0 \% & \Leftrightarrow & \mathrm{C}=\mathrm{C}_{\mathrm{EOL}}=0.8 \times \mathrm{C}_{\mathrm{IV}}
\end{array}
$$

with $\mathrm{C}_{\mathrm{IV}}$ is the initial value of the capacity, and $\mathrm{C}_{\mathrm{EOL}}$ is the value of the capacity at the end of life. $\mathrm{SOH}$ equation based on the capacity [17] is:

$$
S O H=\left(\frac{\frac{C}{C_{I V}}-0.8}{0.2}\right) \times 100 \%
$$

and

$$
C=\triangle S O C * Q
$$

with $\mathrm{C}$ is the value of computed capacity, $\triangle S O C$ is the difference between initial SOC and SOC at the current time, and $\mathrm{Q}$ is the new battery capacity. C was computed with two methods, coulomb counting method and open circuit voltage method respectively. The flowchart of SOH estimation based on the battery capacity is shown in Figure 16. The result of the $\mathrm{C}$ value is shown in Figure 17. As shown in Figure 17, the capacity of a new battery is no more than $2.2 \mathrm{Ah}$ but more than $1.78 \mathrm{Ah}$. That happens because the used value is battery capacity at $80 \%$ SOC. Figure 17 also shows the $\mathrm{SOH}$ change for both methods.

Both methods show similar trends of $\mathrm{SOH}$. The $\mathrm{SOH}$ which is based on coulomb counting method is smaller than the $\mathrm{SOH}$ which is based on open circuit voltage method so that it reaches the end of life threshold first. Both methods are easy to use in estimating $\mathrm{SOH}$, but usually need longer computing time until all data is computed 


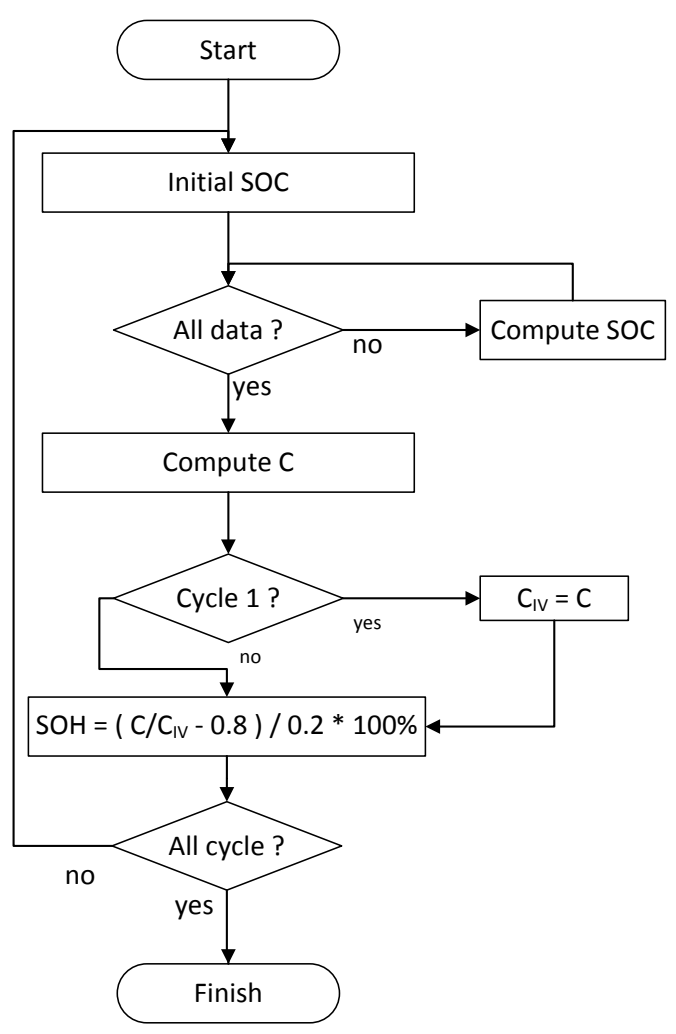

Figure 16. Flowchart of $\mathrm{SOH}$ estimation based on capacity

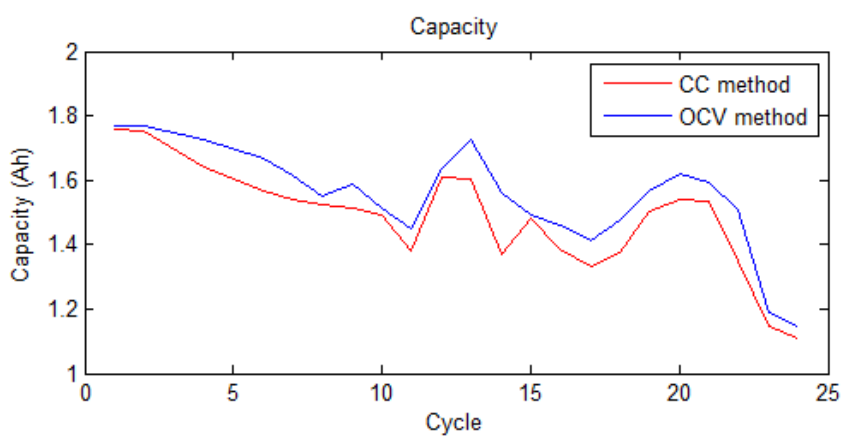

(a)

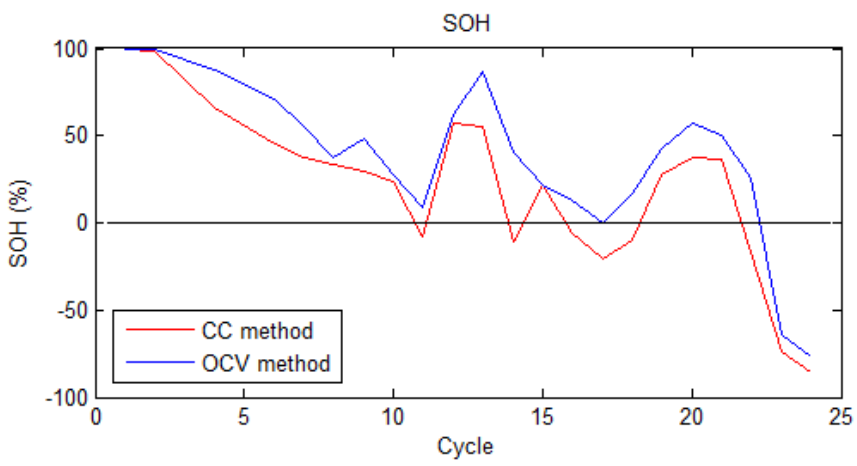

(b)

Figure 17. Change of battery capacity; (a) Capacity - Cycle; (b) SOH - Cycle

\section{Conclusion}

Based on the simulation and experimental results, it can be concluded that the model can be used to estimate the $\mathrm{SOH}$ as proven. The $\mathrm{SOH}$ estimation method based on battery capacity is more accurate than that based on internal ohmic resistance. However, the $\mathrm{SOH}$ estimation method based on internal ohmic resistance is faster. The methods also confirm that the $\mathrm{SOH}$ of the battery decreases according to the increasing number of charge-discharge cycles. 


\section{ACKNOWLEDGEMENT}

This research program is fully supported by Universitas Gadjah Mada Faculty of Engineering. Therefore, the authors would like to express their sincere appreciation to the Faculty for providing the grant.

\section{REFERENCES}

[1] S. Cole and M. Leslie, "Long-term global warming trend sustained in 2013," 2014. [Online]. Available: http://climate.nasa.gov/news/1029/.

[2] A. Dinger et al., "Focus Batteries for Electric Cars," 2010.

[3] T. Sar et al., "A Hybrid Battery Model and State of Health Estimation Method for Lithium-Ion Batteries," pp. 1349-1356, 2014.

[4] S. Li et al., "Study of Battery Modeling using Mathematical and Circuit Oriented Approaches," pp. 1-8, 2011.

[5] H. Lin et al., "Estimation of Battery State of Health Using Probabilistic Neural Network," vol. 9, no. 2, pp. 679-685, 2013.

[6] D. Andre et al., "Comparative study of a structured neural network and an extended Kalman filter for state of health determination of lithium-ion batteries in hybrid electric vehicles," Eng. Appl. Artif. Intell., vol. 26, no. 3, pp. 951-961, 2013.

[7] P. Singh and D. Reisner, "Fuzzy logic-based state-of-health determination of lead acid batteries," 24th Annu. Int. Telecommun. Energy Conf., pp. 583-590.

[8] V. Klass and M. Behm, "A support vector machine-based state-of-health estimation method for lithium-ion batteries under electric vehicle operation," vol. 270, pp. 262-272, 2014.

[9] Z. Guo et al., "State of health estimation for lithium ion batteries based on charging curves," J. Power Sources, vol. 249, pp. 457-462, 2014.

[10] Y. Zou et al., "Combined State of Charge and State of Health estimation over lithiumion battery cell cycle lifespan for electric vehicles," J. Power Sources, vol. 273, pp. 793-803, 2015.

[11] C. R. Gould et al., "New Battery Model and State-of-Health Determination Through Subspace Parameter Estimation and StateObserver Techniques," vol. 58, no. 8, pp. 3905-3916, 2009.

[12] A. Hentunen et al., "Electrical battery model for dynamic simulations of hybrid electric vehicles," 2011 IEEE Veh. Power Propuls. Conf., pp. 1-6, Sep. 2011.

[13] X. Li and S. Choe, "State-of-charge (SOC) estimation based on a reduced order electrochemical thermal model and extended Kalman filter," $2013 \mathrm{Am}$. Control Conf., pp. 1100-1105, Jun. 2013.

[14] X. Hu et al., "A comparative study of equivalent circuit models for Li-ion batteries," J. Power Sources, vol. 198, pp. 359-367, 2012.

[15] L. Lu et al., "A review on the key issues for lithium-ion battery management in electric vehicles," J. Power Sources, vol. 226, pp. 272-288, Mar. 2013.

[16] A. Hand, "No Title." [Online]. Available: http://www.hobbyking.com/hobbyking/store I_317_85_Batteries_AccessoriesTurnigy_Lipoly.html. [Accessed: 20-Nov2015].

[17] A. Zenati et al., "A Methodology to Assess the State Of Health of Lithium-ion Batteries Based on the Battery' s Parameters and a Fuzzy Logic System," IECON 2010, 2010.

[18] E. Davis et al., "Internal ohmic measurements and their relationship to battery capacity - epri's ongoing technology evaluation," pp. 1-10.

[19] M. U. Cuma and T. Koroglu, "A comprehensive review on estimation strategies used in hybrid and battery electric vehicles," Renew. Sustain. Energy Rev., vol. 42, pp. 517-531, 2015.

[20] J. Remmlinger et al., "On-board state-ofhealth monitoring of lithium-ion batteries using linear parameter-varying models q," $J$. Power Sources, vol. 239, pp. 689-695, 2013. 